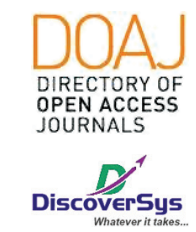

Published by DiscoverSys

\section{Diabetes insipidus after suprasellar tumor surgery: Case report}

\author{
Yuriz Bakhtiar, ${ }^{1}$ Muhamad Thohar Arifin, ${ }^{1 *}$ Adrian Pratama, ${ }^{2}$ Jacob Bunyamin ${ }^{1}$
}

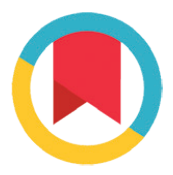

CrossMark

\title{
ABSTRACT
}

Introduction: Diabetes insipidus (DI) is a rare water and electrolyte imbalance with hypovolemic hypernatremia characteristic (1:25.000) however can be potentially fatal if not well-anticipated and managed adequately. Brain tumors, either operated or not, may lead to DI (25\%). Based on anatomical location, the suprasellar tumor may cause hypophyseal hormone dysfunction. Sellar region tumors can be classified into hypophyseal (90\%) and non-hypophyseal (10\%). Nonhypophyseal tumors with neurological deficits are more often causing hypophyseal hormone hyposecretion including DI. Sellar tubercle meningioma is one of the sellar region non-hypophyseal tumors which is often encountered and surgically managed. Worsening symptoms may become the surgical indication e.g. headache and visual disturbance.
Case: We are reporting two meningioma cases in Salatiga local hospital with almost similar tumor characteristics either size and frontal lobe location. Both cases were managed by similar transcranial tumor excision craniotomy. Their differences were in the presence of neurological manifestations and the degree of hypothalamic compression. One patient developed postoperative DI without thirst thus the clinical manifestation was polyuria which was closely monitored in the intensive ward. We were convinced that the DI incidence was affected by the tumor manifestation and location which can be assessed by computed tomography (CT) scans.

Conclusion: The clinicians should anticipate the possibility of post craniotomy DI thus may provide adequate monitoring and management in patients developing postoperative DI.
'Department of Neurosurgery, Faculty of Medicine, Universitas Diponegoro-Kariadi General Hospital, Semarang, Indonesia 2Department of Neurosurgery, Salatiga City Hospital, Indonesia

*Correspondence to:

Muhamad Thohar Arifin, Department of Neurosurgery, Faculty of Medicine, Universitas DiponegoroKariadi General Hospital, Semarang, Indonesia

thohar@fk.undip.ac.id

Received: 2020-07-27 Accepted: 2020-06-29 Published: 2020-08-01
Keywords: diabetes insipidus, post-craniotomy, meningioma

Cite This Article: Bakhtiar, Y., Arifin, M.T., Pratama, A., Bunyamin, J. 2020. Diabetes insipidus after suprasellar tumor surgery: Case report. Intisari Sains Medis 11(2): 722-727. D0I: 10.15562/ism.v11i2.807

\section{INTRODUCTION}

Diabetes insipidus (DI) is a disorder of water and electrolyte imbalance characterized by hypernatremia accompanied by a physiological inability to produce concentrated urine leading to enormous loss of free water molecules and subsequently dehydration (hypernatremic hypovolemia). ${ }^{1,2} \mathrm{DI}$ is often found in hypophyseal tumors followed by hypothalamic and Rathke pouch-invading tumors. ${ }^{3,4}$ The prevalence of DI is quite rare i.e. less than 1:25,000 cases and a quarter of it is observed in the central nervous system (CNS) tumor cases or the presence of prior tumor surgery. ${ }^{5}$

CNS injury either caused by trauma or surgery is known to induce the development of DI. ${ }^{4}$ The exact mechanism of this phenomenon is not understood. However, hypothalamic-hypophysis area necrosis, infarction, or hemorrhage may contribute to the dysfunction of hypophyseal hormones including DI. ${ }^{4}$ The prevalence of postoperative DI may reach $4.4 \%$ in non-hypophyseal tumors after transcranial surgery. ${ }^{4}$ Although considered rare, postoperative DI may lead to death after transcranial surgery either in the pediatric or adult population. ${ }^{1}$ The high mortality is caused by the dehydration process due to polyuria leading to hemodynamic imbalance and organ perfusion failure. ${ }^{1,3}$ Furthermore, the accompanying hypernatremia is also responsible in the altered state of consciousness (lethargy and irritability) and status epilepticus which could terminate in death. ${ }^{1}$

We are reporting two cases of DI in two CNS tumor patients in the frontal lobe extending to the suprasellar area admitted to Salatiga City Hospital, Indonesia. The clinical presentation was slightly distinct, with a unilateral headache more dominantly reported by one patient and visual disturbance in the other. Brain computed tomography (CT) scan findings demonstrated similar tumor morphology. However, they differed in location as one patient had a right frontal lobe extending to suprasellar region pushing the contralateral hemisphere. In contrast, the other had a mid-frontal tumor extending to suprasellar region and pushing the third ventricle. We performed tumor excision craniotomy to both, however one patient developed postoperative DI which was regularly monitored for urine output and serum sodium levels. We are comparing both cases to investigate the correlation between non-hypophyseal tumor location to the postoperative DI possibility to enrich the literature in this field and to increase the clinicians' awareness of post-transcranial brain surgery DI which might fatally harm the patient. 


\section{CASE REPORTS}

\section{Case 1}

A 50-year old lady was admitted due to chronic progressive headache since a month before admission which was worsened at night. She also complained of double vision, denied projectile vomiting and seizure, and admitted a history of hormonal contraception use. General physical and
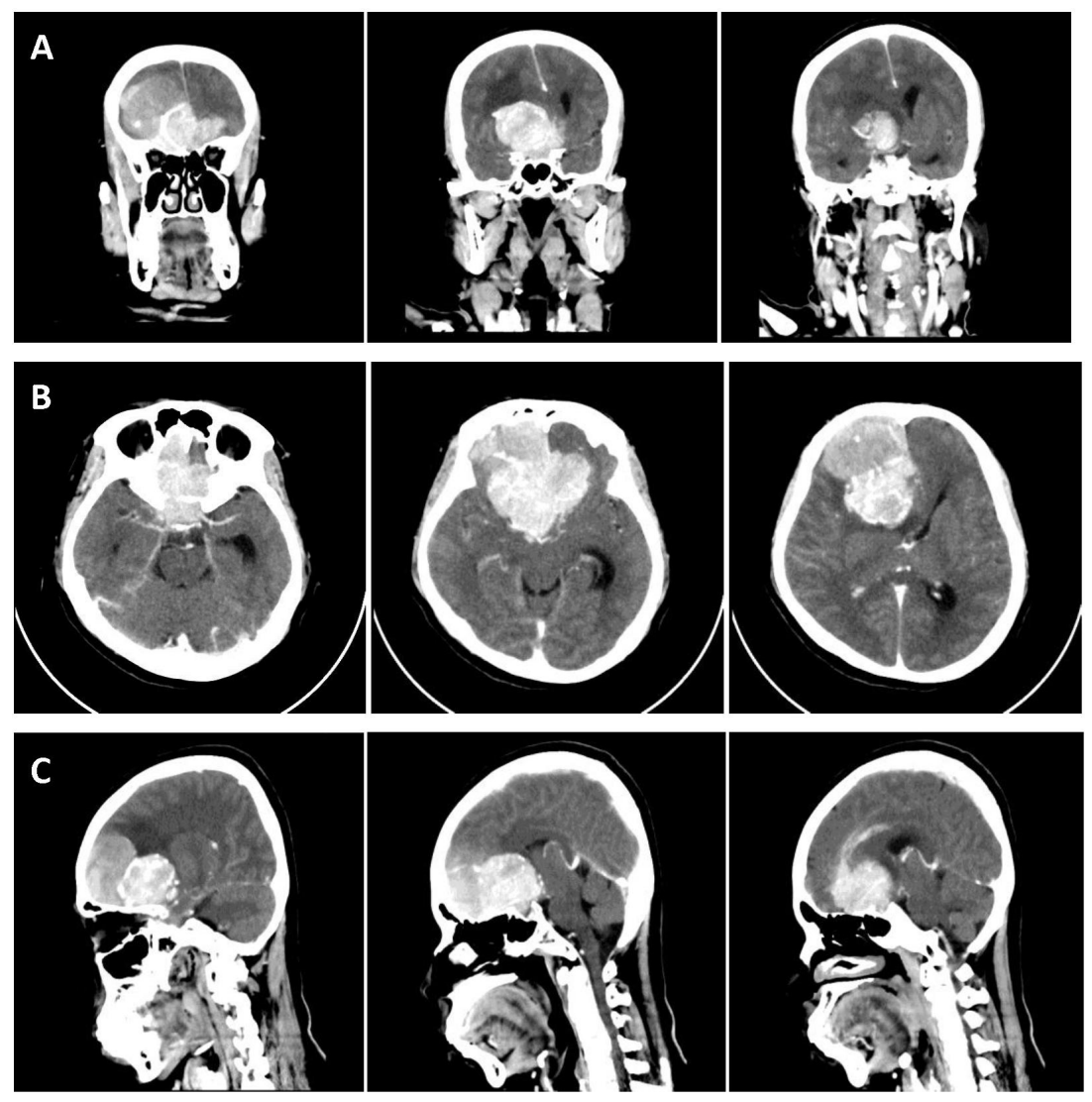

Figure 1 Contrast-enhanced a.) coronal b.) axial, dan c.) sagittal CT sections showing bilateral parasagittal meningioma extending to skull base with left subfalcine herniation. The mass appeared to occupy the right hypothalamic area

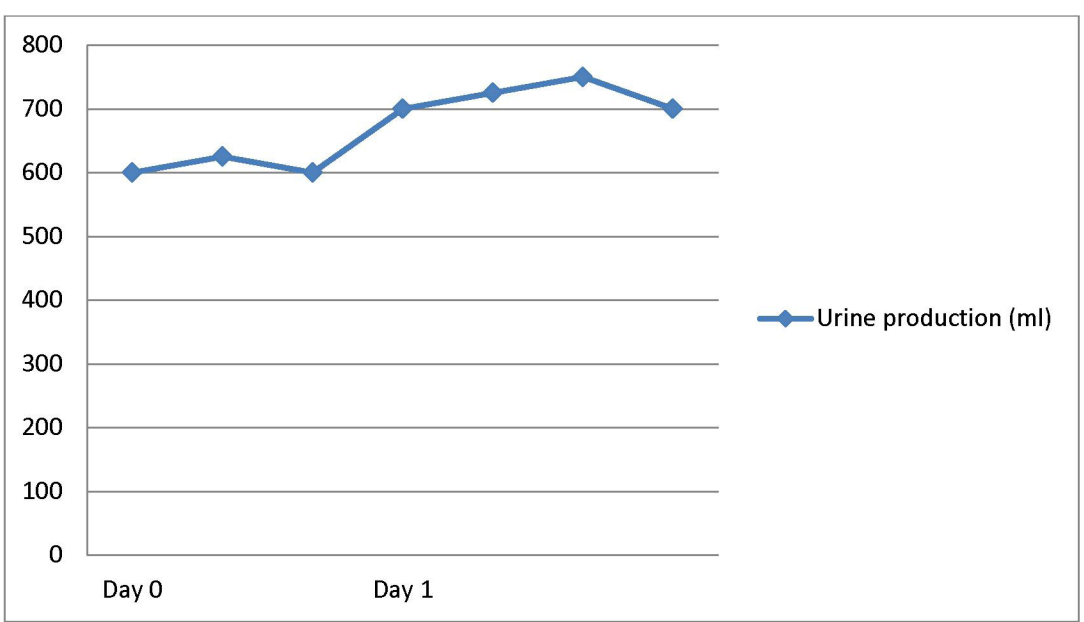

Graph 1 Postoperative urine production in patient 1 neurological examinations were normal. Imaging showed an intracranial mass suspected as bilateral parasagittal meningioma with subfalcine herniation $\pm 13 \mathrm{~mm}$ to the left (figure 1). A lumbar drain was planned followed by tumor excision craniotomy. Preoperative dexamethasone was administered to reduce the vasogenic edema. Urine production was monitored (graph 1) without signs of polyuria and thirst complaints. A follow-up CT scan did not demonstrate signs of increased intracranial pressure (ICP) and hemorrhage. The pathological result confirmed transitional meningioma. She performed well postoperative and reported reduced headache. and then discharged after 15 days.

\section{Case 2}

A 43-year old lady was admitted due to blurring of vision since the year before which progressed within the last 3 months. She complained of mild headache, denied projectile vomiting and seizure, and admitted a history of hormonal contraception use. General physical examination was normal, however, her visual acuity was reduced to $<1 / 60$ in bilateral eyes. She was then diagnosed with retrobulbar neuritis and sent for imaging examination. CT scan demonstrated an intracranial mass suspected as malignancy in frontobasal area (figure 2). She was scheduled for tumor excision craniotomy and received dexamethasone premedication. Postoperatively she developed diabetes insipidus with serum sodium level $150 \mathrm{mEq} / \mathrm{L}$, 6 hours urine production $2,850 \mathrm{ml}$, and monitored intensively (graph 2 and 3). We could not measure urine osmolality due to facility limitations. She then treated with a regiment of vasopressin injection 5 units q.i.d and desmopressin nasal spray 2 puffs b.i.d which was tapered down based on clinical response (graph 2). During the intensive observation, she managed to maintain clear consciousness, hemodynamically stable and did not complain of thirst. Serum sodium level and urine production improved in day-3 post-surgery and she was then discharged on day- 6 with improved visual acuity to $2 / 60$ bilaterally under desmopressin spray. The pathological examination resulted in psammomatous meningioma.

\section{DISCUSSION}

Based on its origin, suprasellar mass or tumor can be classified into hypophyseal (90\%) and nonhypophyseal (10\%). ${ }^{6}$ Non-hypophyseal tumors often cause hypophyseal hormones hyposecretion including DI although it is rare and accompanied with a dominant neurological deficit due to mass effect lesion. ${ }^{6}$ Common neurological complaints including headache, visual disturbance, cranial 

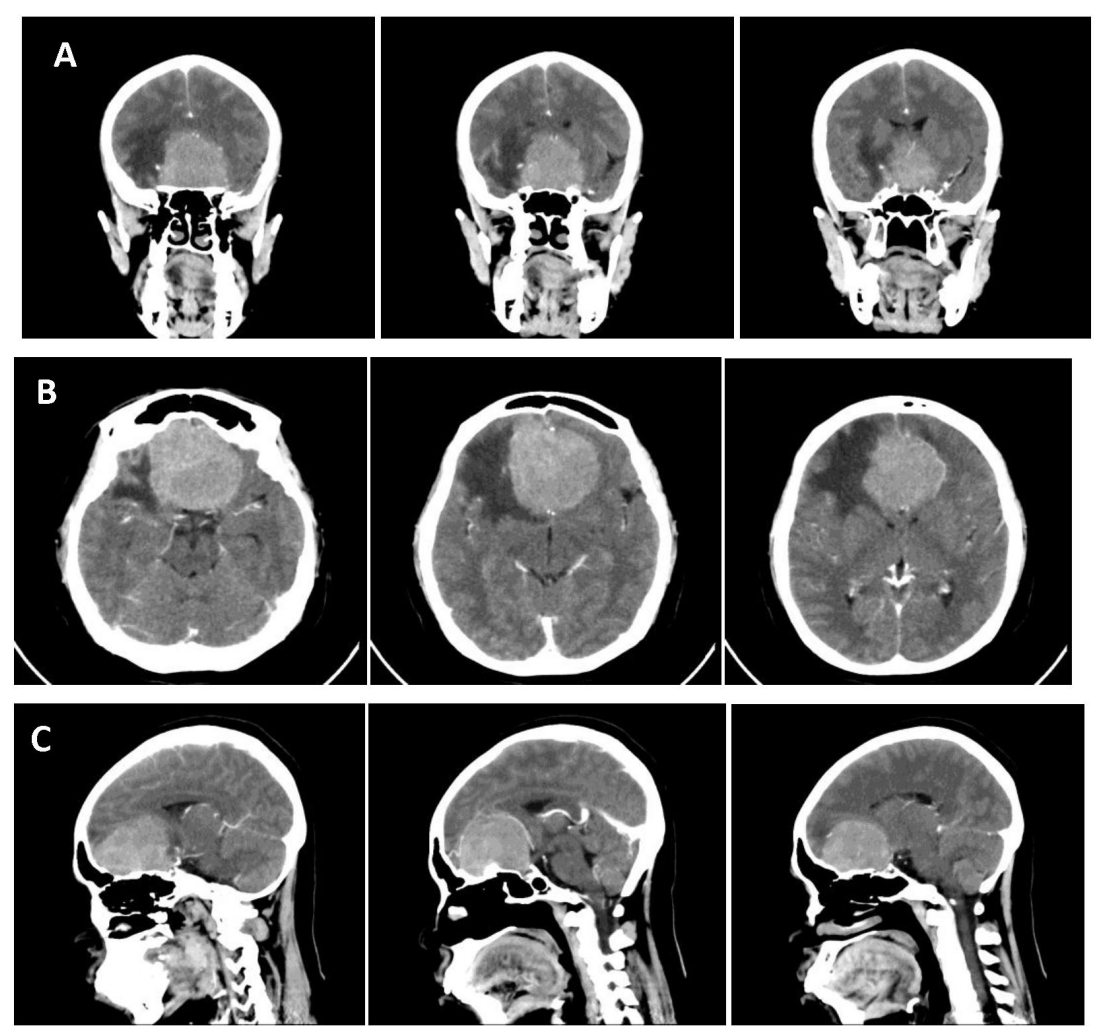

Figure 2 Contrast-enhanced a.) coronal, b.) axial, dan c.) sagittal CT sections showing intracranial mass suspected to be malignant in frontobasal region accompanied by vasogenic edema. The bilateral hypothalamus was seen shifted posteriorly due to the mass

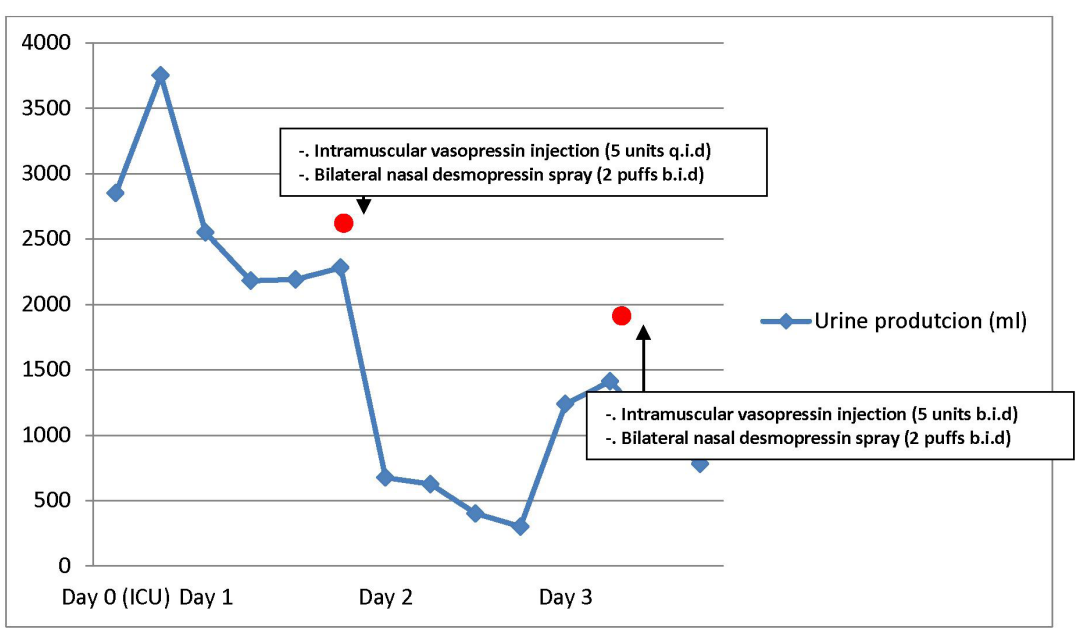

Graph 2 Postoperative urine production in patient 2

nerves neuropathy, hydrocephalus, and altered state of consciousness. ${ }^{6}$ Meningioma should be considered as a differential diagnosis in suprasellar mass cases especially sellar tubercle meningioma which often causes visual loss due to pressure in optic nerves or chiasm..$^{6-8}$ Our second patient complained of dominant visual loss rather than headache although the radiological findings were almost similar i.e. intracranial mass pushing the optic canal and extend to the optic chiasm. Due to
CT limitations, it was still unclear why she developed a more pronounced visual loss. Therefore, we would suggest that a more advanced imaging e.g. Magnetic Resonance Imaging (MRI) should be performed as it can give details of the anatomical structure for both cases, as the mass may origin from the sellar tubercle thus the risk of developing DI will be higher. ${ }^{6}$

Central or neurogenic DI may be caused by the antidiuretic hormone $(\mathrm{ADH})$ or arginine vasopressin (AVP) deficiency in the hypothalamichypophyseal axis (HHA) disturbance which is often found in suprasellar mass, post-CNS surgery, congenital lesions, head trauma, and inflammation., Arginine vasopressin is a non-peptide secreted the supraoptic and paraventricular hippocampal nucleus transmitted by the medial eminence to the neurohypophysis to be metabolized to its active form. ${ }^{10,11}$ Its deficiency may be found in primary hypothalamic lesions or secondary due to mass effect. ${ }^{11}$ Hypothalamus secretes AVP depending on the plasma osmolality state and aims to maintain its homeostatic level $(280-290 \mathrm{mOsm} / \mathrm{kg}){ }^{11}$ If the plasma osmolality exceeds $290 \mathrm{mOsm} / \mathrm{kg}$, AVP will be secreted and the thirst receptor in hypothalamus will be activated to return to the normal level. ${ }^{11}$

The clinical manifestation of DI usually arises if the magnocellular neurons damage in the hypothalamus reaches $80-90 \%$, which $50-60 \%$ of it is caused due to distal hypothalamic-hypophyseal axis damage post-surgery. ${ }^{10}$ Diagnostic criteria of postoperative DI can be established based on (1) surgery on hypothalamus-hypophyseal axis (hypophyseal gland, infundibulum stalk, and hypothalamus) either in trans-sphenoid or trans-cranial approach, (2) polyuria with urine volume $>3 \mathrm{ml} / \mathrm{kg}$ BW/hours within 2 hours post-surgery, (3) hypotonic urine with osmolality $<300 \mathrm{mOsm} / \mathrm{kg}$ BW and/or specific gravity $<1.010$, (4) hypernatremia, which the sodium level $>145 \mathrm{mEq} / \mathrm{L}$, and (5) increase of plasma osmolality $>300 \mathrm{mOsm} / \mathrm{kg} \mathrm{BW.} .^{1,2}$ These criteria were used to diagnose postoperative DI in patient 2, as the tumor region was located in the suprasellar region adjacent to the hypothalamus, development of polyuria up to 2,850 ml with 2 hours post-surgery, and the serum sodium level reaching $150 \mathrm{mEq} / \mathrm{L}$. However, we were unable to measure urine and plasma osmolality due to limited facilities in the local hospital.

MRI can demonstrate better anatomical structure visualization however due to facility limitation, we were only able to perform head CT scan. Postoperative DI incidence may reach 30\% which is dependent on the third ventricle and hypothalamus deformity. ${ }^{3}$ Based on MRI appearance, this deformity can be classified into three stages i.e. 


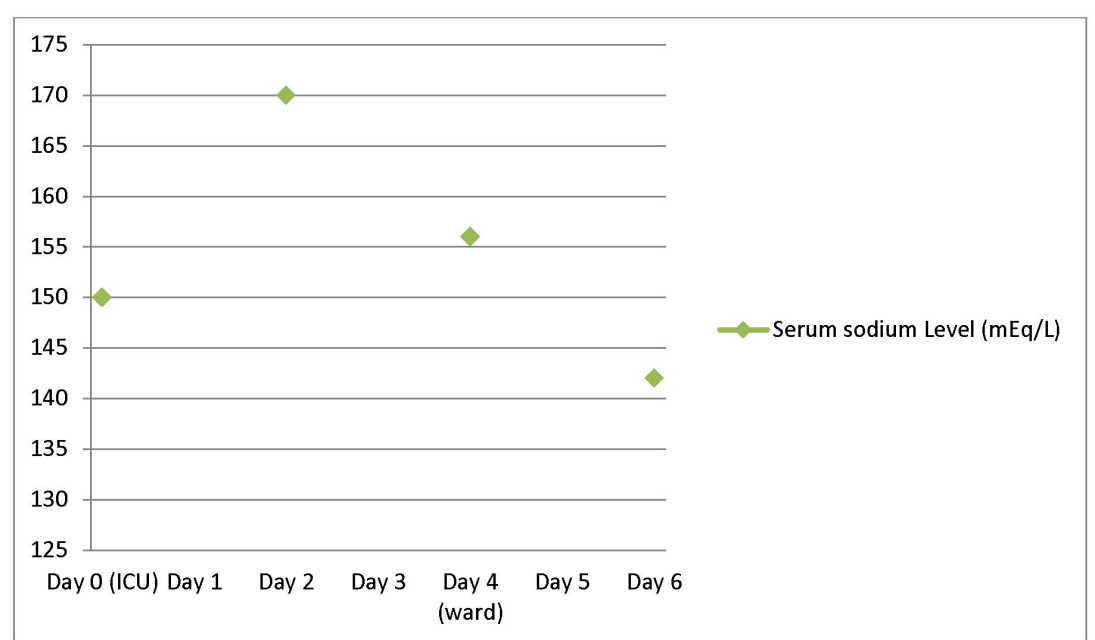

Graph 3 The serum sodium level in patient 2
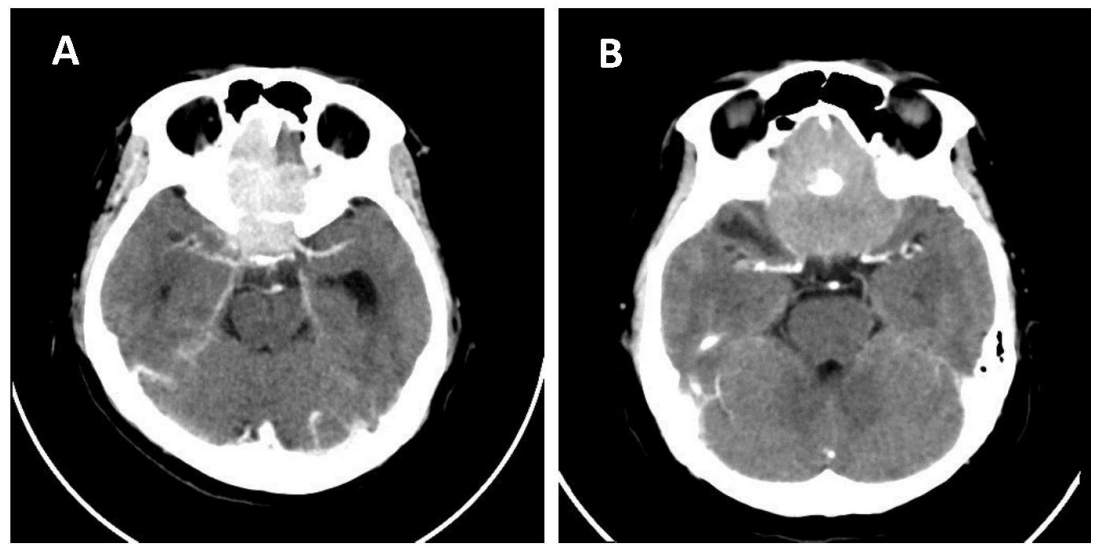

Figure 3 Contrast-enhanced axial slices CT in a) patient $1 \mathrm{~b}$ ) patient 2 showing a similar mass in frontobasal area extending to the optic canal and chiasm

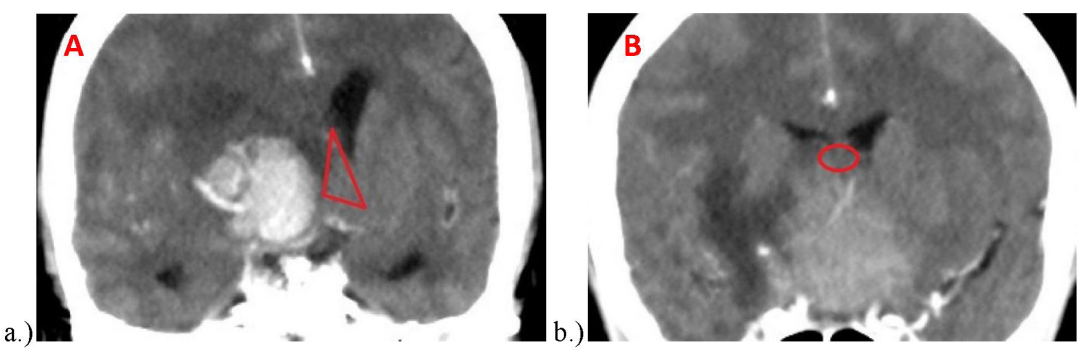

Figure 4 Contrast-enhanced coronal section of a) patient 1 where the left hypothalamus (red triangle) is slightly present and b) patient 2 where the hypothalamus region disappeared (red circle showed the estimation of the compressed hypothalamus)

stage 0 (clear visualization of the third ventricle and hypothalamus), stage 1 (compressed third ventricle and missing hypothalamus, however, the ventricle is clearly observed), and stage 2 (missing third ventricle and hypothalamus either accompanied with hydrocephalus or not). ${ }^{3}$ We compared the deformity based on the $\mathrm{CT}$ images and the patient
1 demonstrated missing third ventricle and right hypothalamus structure although the left hypothalamus is still visualized (Figure 4a). On the contrary, in patient 2 all third ventricle and hypothalamus regions were missing (Figure $4 \mathrm{~b}$ ). Based on the severity of hypothalamus compression which reached stage 2 involving the bilateral hypothalamus we were convinced that the postoperative DI risk was greater in patient 2 .

Continuous thirst leading to polydipsia may become a subjective complaint besides polyuria in DI patients. ${ }^{1,2,12}$ Interestingly, patient 2 did not complain of thirst and she often declined when ordered to drink. This phenomenon may be explained due to disturbance in the hypothalamic thirst center. ${ }^{11,12}$ The thirst center in the hypothalamus is composed of subfornical organs located in the ventral surface of fornix near to the foramen of Monroe, organum vasculosum, and lamina terminalis which is the anterior border of the hypothalamus (Figure 5a). ${ }^{12,13}$ We thought that she did not develop polydipsia due to the compression of the anterior hypothalamus. (Figure 5b) This needs to be concerned as the absence of thirst may worsen the dehydration as the urine volume increases excessively without compensated by adequate water intake.

We did not observe the triphasic pattern of serum sodium level in our patient, which is quite rare in adults affecting $1.1 \%$ of adult patients and $22 \%$ of pediatric patients. ${ }^{14,15}$ The patient 2's urine production demonstrated the triphasic pattern with two polyuric phases. The first polyuric phase represents hypothalamic dysfunction, followed by an oliguric phase because of disturbance in vasopressin secretion caused by hypothalamic trauma due to tumor's effect and surgery thus the secretion of vasopressin becomes impaired and causing the second polyuric phase. ${ }^{14}$ Continuous monitoring of urine production is essential to avoid worsening in this second polyuric phase. This phase can also be present when the serum sodium level drops below the normal range which is often happened in the day- 6 or day- 9 after surgery, although in our case the patient developed it in day-3. ${ }^{14}$

Based on surgery types, Baysefer et al. reported that the DI incidence reached 50\% after suprasellar meningioma transcranial excision. ${ }^{16}$ More recently, due to medical technology advances, the postoperative DI rate in suprasellar meningioma excision was reported as $4.1-7 \%$ either conducted in bicoronal or sub frontal approach. ${ }^{17,18}$ From this fact, we can see that the transcranial surgery procedure always bears the risk of DI development if the case seen is a suprasellar meningioma. 


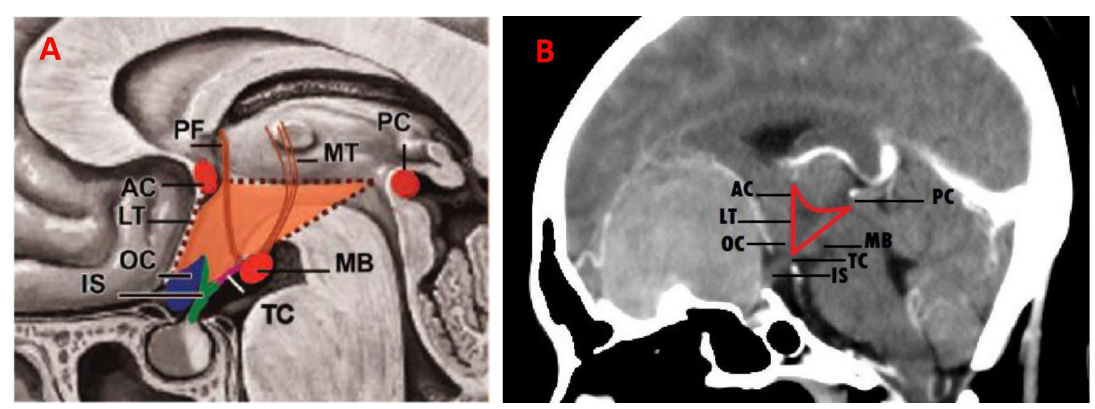

Figure 5 a.) Hypothalamus location (red triangle) which is inferior to an imaginary line connecting anterior commissure $(\mathrm{AC})$ and posterior commissure (PC), posterior of lamina terminalis (LT), and anterior of the imaginary line connecting mammillary bodies (MB) and posterior commissure (PC). ${ }^{13}$ b.) Contrast-enhanced CT in the sagittal section of patient 2 demonstrated the tumoral mass effect pushing the lamina terminalis posteriorly which may prevent thirst in $\mathrm{DI} . \mathrm{AC}=$ anterior commissure, $\mathrm{PC}=$ posterior commissure, $\mathrm{MB}=$ mammillary bodies, $\mathrm{TC}=$ tuber cinereum, $\mathrm{IS}=$ infundibulum stalk, OC = optic chiasm, LT = lamina terminalis

\section{CONCLUSION}

Diabetes insipidus is one of the complications after suprasellar tumor surgery which is rarely seen. However, it can be fatal if remains undetected and not adequately managed. Patients with sellar tubercle meningioma often demonstrated visual disturbances symptom and more potentially affected by hyposecretion of hypophyseal hormones including DI. MRI should be a preferred radiological examination as it enables clinicians to evaluate the anatomical structure of the hypothalamus-hypophysis axis to anticipate the occurrence of postoperative DI.

The radiological appearance of the compressed third ventricle and bilateral hypothalamus may increase the risk of postoperative DI. Thirst response is often disturbed in hypothalamus tumor cases leading to potentially fatal dehydration in the polyuric phase. Close urine and serum sodium monitoring must be performed to reduce the dehydration risk in the second polyuric phase. All transcranial surgery with either bicoronal or sub frontal approach still bears the $4.1-7 \%$ risk of postoperative DI, thus the clinician needs to anticipate this risk, especially in suprasellar meningioma cases if neurological deficits e.g. visual disturbance present and the tumor location compressing hypothalamus.

\section{DECLARATION OF COMPETING INTEREST}

We declare no conflict of interest.

\section{SOURCES OF FUNDING}

Nothing to declare.

\section{CONSENT}

Written informed consent was obtained from the deceased patient's family. Information within the paper has been sufficiently anonymized not to cause harm to the patient or their family. A copy of a signed document stating this is available for review by the Editor-in-Chief of this journal on request.

\section{AUTHOR CONTRIBUTION}

Yuriz Bakhtiar:

Performed surgery, conception of report, data collection, data analysis, manuscript writing, revision.

Muhamad Thohar Arifin: Conception of report, data analysis, and manuscript revision and submission.

Adrian Pratama:

Data analysis, manuscript writing and manuscript revision.

Jacob Bunyamin:

Data analysis, manuscript writing and manuscript revision.

\section{REFERENCES}

1. Winn RH. Youmans \& Winn Neurological Surgery. 7th edition. Philadelphia: Elsevier; 2017.

2. Greenberg MS. Handbook of Neurosurgery. 8th edition. New York: Thieme; 2016.

3. He J, Jia G, Jia W, Li D, Ni M, Wang S, et al. Clinical Predictors of Diabetes Insipidus After Transcranial Surgery for Pituitary Adenoma. World Neurosurg. 2017; 101:1-10.

4. Aimaretti G, Corneli G, Croce CG, Ghigo E, Grottoli S, Rovere $S$, et al. Endocrine dysfunction in patients operated on for non-pituitary intracranial tumors. European Journal of Endocrinology. 2006; 155:559-566.

5. Das K, Joseph F, Khan M, Mon A, Sharma D, Sinha A, et al. Cranial diabetes insipidus with pituitary stalk lesion. QJM: An International Journal of Medicine. 2016; 109:703-8.

6. Bronstein MD, Glezer A, Paraiba DB. Rare Sellar Lesions. Endocrinol Metab Clin N Am. 2008; 37:195-211.

7. Dotan S, Mizrahi CJ, Mosovici S, Spektor S. Optic Nerve Vascular Compresion in a Patient with a Tubercullum Sellae Meningioma. Case Reports in Ophthalmological Medicine 2015.

8. Chai Y, Kondo A, Oshitari T, Yamamoto S, Yamazaki H. Case of acute optic nerve compression caused by tubercullum sellae meningioma with optic canal involvement. Clinical Ophthalmology. 2012; 6:661-6.

9. Mikoś H, Moszyńska MO, Niedziela M, Perek D. Diabetes insipidus coexisting with sellar-suprasellar tumor- case report. Wspolczesna Onkol. 2011;15(6):397-400.

10. Chowdhury S, Kalra S, Jain SM, Malve H, Thakkar PB, Thomas N, et al. Diabetes insipidus: The other diabetes. Indian J Endocr Metab. 2016;20:9-21.

11. Lamas C, Pozo C, Villabona C. Clinical guidelines for management of diabetes insipidus and syndrome of inappropriate antidiuretic hormone secretion after pituitary surgery. Endocrinol Nutr. 2014;61:15-24. 
12. Barger GR, Modawi I, Rossi NF. Central diabetes insipidus and adipsia due to astrocytoma: diagnosis and management. CEN Case Rep. 2013;2:11-6.

13. Lee DH, Saleem SN, Said AHM. Lesions of the Hypothalamus: MR Imaging Diagnostic Features. Radio Graphics. 2007;27: 1087-1108.

14. Hoorn EJ, Zietse R. Water balance disorders after neurosurgery: the triphasic response revisited. NDT Plus. 2010;3:42-4.

15. Boot AM, Claahsen-van der Grinten HL, Finken MJJ, Han K, Hoving EW, Kruis RWJ, et al. Management and consequences of postoperative fluctuations in plasma sodium concentration after pediatric brain tumor surgery in the sellar regions: a national cohort analysis. Pituitary 2018;21:384-392.

16. Baysefer A, Erdogan E, Gezen F, Timurkaynak E, Seber N. Surgical Treatment of Sellar Regions Neoplasms. Turkish Neurosurgery. 1997;7:1-4
17. Hamada S, Nabih A, Nosseir M, Osman M, Saoud K, Wild A. Evaluation of Tumor Resection and Optic Aparatus Decompression in Cases of Supra-sellar and Parasellar Meningioma Using Two Different Surgical Modalities: A Comparative Studies. EC Neurology. 2019;11:949-957.

18. Cheng XB, Lu ZF, Shi BZ, Zhao YG. Twenty-nine cases of resection of suprasellar meningioma through small bone window: an interhemispheric approach. Contemp Oncol. 2013;17(6):525-9.

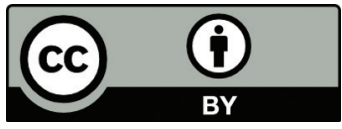

This work is licensed under a Creative Commons Attribution 